\title{
Electron Density and Dielectric Properties of highly porous MOFs
}

\author{
Rebecca Scatena $^{1}$, Yannick Thomas Guntern ${ }^{1}$, Piero Macchi ${ }^{1}$ \\ ${ }^{1}$ University Of Bern, Department Of Chemistry And Biochemistry, Bern, Switzerland \\ E-mail: rebecca.scatena@dcb.unibe.ch
}

We have investigated the correlation between the accurate electron density (ED) distribution and the dielectric properties of highly porous metal organic frameworks (MOFs). Recently, these materials have attracted interest because the combination of a framework structure and large voids represents a promising route to develop new low-dielectric constant ( $\varepsilon r$ ) materials, retaining the required thermal stability, high mechanical strength and good adhesion to neighboring layers[1]. Among the known porous MOFs, [Cu3(BTC)2]n (BTC=benzene-1,3,5-tricarboxylate) and the isostructural [Zn3(BTC)2]n have been selected as they combine porosity, high structural symmetry, high single crystal quality and thermal stability up to $240^{\circ} \mathrm{C}[2]$. This class of isostructural compounds allows to investigate the effect of different metal ions and of guest molecules on the Er magnitude and frequency dependence. In this work, the possibility to prepare an empty MOF structure and to study its dielectric properties have been examined to improve the selection of promising low- $\varepsilon r$ interlayer materials. Different desolvation procedures have been tested on single crystal defining the desolvation limits and stability, and improving the data quality of high resolution X-ray measurements for accurate ED determination. In addition, virtual desolvation algorithms have been adopted and examined to remove the contribution of the solvent to the diffraction. This procedure is unprecedented for high resolution data employed in ED modelling. The goal was monitoring the effect of the solvent on the electron density distribution, for example by comparing the theoretical simulations against experimental models for crystals with different levels of desolvation. Estimates of the static $\mathrm{Er}$ for periodic systems, as well as frequency dependent ones of selected fragments of the frameworks can be obtained from density functional theory (DFT). A periodic wavefunction does not address directly which functional group/building block of a system contributes more to a specific property. Therefore, their specific contributions to the $\varepsilon r$ were calculated quantum mechanically on selected fragments in gas phase. This has also allowed to interpret the stereo-electronic differences induced by different metals[3]. The joint analysis of theoretical and experimental data was crucial for an interpretation of the experimental results at an atomic and molecular level. The purpose of this research is to identify the molecular or sub-molecular features that impart the $\varepsilon r$ for a material design development. To the best of our knowledge, this study represents the first systematic investigation of the correlation between dielectric constant and electron density distribution in MOFs. In a long term view, we aim to predict materials properties from the electron density of their smallest building blocks, molecules or functional groups.

[1] Usman, M. et al. (2015) ChemElectroChem. 6, 786-788.

[2] Liu, YD. et al. (2012) ChemCommun(Camb). 45, 5635-5637.

[3] Ernst, M. et al. (2016) CrystEngComm. 18, 7339-7346.

Keywords: electron density, dielectric properties, porous metal organic frameworks 